\title{
The value of the Barcelona Clinic Liver Cancer and alpha-fetoprotein in the prognosis of hepatocellular carcinoma
}

\author{
Rafael Gómez-Rodríguez ${ }^{1}$, Marta Romero-Gutiérrez ${ }^{1}$, Tomás Artaza-Varasa ${ }^{1}$, \\ Concepción González-Frutos ${ }^{1}$, Juan José Ciampi-Dopazo ${ }^{2}$, Gema-de-la-Cruz-Pérez ${ }^{1}$ \\ and Juan José Sánchez-Ruano ${ }^{1}$
}

${ }^{1}$ Unit of Hepatology. Department of Gastroenterology. ${ }^{2}$ Unit of Interventional Radiology. Hospital Virgen de la Salud. Toledo, Spain

\begin{abstract}
Introduction and objectives: presently, the reference staging system to evaluate the prognosis of hepatocellular carcinoma (HCC) patients is "The Barcelona Clinic Liver Cancer" (BCLC) system. The value of alpha-fetoprotein (AFP) has not been properly defined. The aim of this study was to evaluate the BCLC classification in our clinical practice and to know what the prognostic value of AFP is.

Material and methods: 136 consecutive $\mathrm{HCC}$ patients were prospectively included in this study. The diagnosis of HCC was based on the recommendation of international guidelines. The patients were studied and managed according to usual clinical practice. Survival curves were estimated using the Kaplan-Meier method and predictors of survival were identified using the Cox model.

Results: 110 patients (80.9\%) were male. The mean age of the patients was $66.62 \pm 11.68$ years. Liver cirrhosis was present in $91.2 \%$. The most frequent cause of liver disease was hepatitis $\mathrm{C}$ infection (38.97\%). Serum AFP was $\leq 20 \mathrm{ng} / \mathrm{mL}$ in the $57 \%$, > $20-200 \mathrm{ng} / \mathrm{mL}$ in the $20 \%$, and $>200 \mathrm{ng} / \mathrm{mL}$ in $23 \%$. According to the BCLC staging system, 79 patients were classified as stage $\mathrm{A}$ (58.09\%), $29(21.32 \%)$ stage B, $17(12.50 \%)$ stage C and 11 patients $(8.09 \%)$ as stage $\mathrm{D}$. The overall median survival time was 26.52 months (95\% CI 16.7-36.3). The median survival according to BCLC system was: BCLC A 62.27 , BCLC B 12.72, BCLC C 4.83 , and BCLC D 0.62 months ( $p<0.0001)$; and according to serum AFP was: AFP $\leq 20$ : 62.27 months, $>20-200: 22.08$ months, and $>200 \mathrm{ng} / \mathrm{mL}: 5.39$ months $(p<0.0001)$. Multivariate analysis showed that AFP, BCLC classification and treatment were independent prognostic factors.

Conclusions: our results confirm that the BCLC is a good prognostic system. The AFP has prognosis value in HCC patients. The addition of AFP could improve the BCLC system. Future studies are needed to confirm our results and also the best way to combine BCLC and AFP properly.
\end{abstract}

Key words: Hepatocellular carcinoma. BCLC staging. Alpha-fetoprotein.

Received: 25-11-2011

Accepted: 02-03-2012

Correspondence: Rafael Gómez-Rodríguez. Department of Gastroenterology. Hospital Virgen de la Salud. Avda. Barber, 30. 45004 Toledo, Spain e-mail:925252585@telefonica.net
Gómez-Rodríguez R, Romero-Gutiérrez M, Artaza-Varasa T, González-Frutos C, Ciampi-Dopazo JJ, de-la-Cruz-Pérez G and Sánchez-Ruano JJ. The value of the Barcelona Clinic Liver Cancer and alpha-fetoprotein in the prognosis of hepatocellular carcinoma. Rev Esp Enferm Dig 2012; 104: 298-304.

\section{INTRODUCTION}

Hepatocellular carcinoma (HCC) is the most common primary liver malignancy (1) and is the major cause of deaths in patients with cirrhosis $(2,3)$. There are regional variations in the mortality rates of HCC $(1,4)$. An increased incidence in southern Europe is probably related to hepatitis $\mathrm{B}$ and $\mathrm{C}$ infection and increasing alcohol intake (5), which are major risk factors for $\operatorname{HCC}(2,6)$.

In a population-based data study, the five-year relative survival ranged from $8 \%$ in the southern Europe to 5\% in Eastern Europe, and 6\% in the United States (5). In Spain the mortality rates from HCC in the years 2000-2004 was 4.92 per 100,000 men and 1.52 per 100,000 women (4).

The surveillance in an increased risk population can reduce HCC mortality (7-9). The American Association for the Study of Liver Diseases (AASLD) recommends surveillance for HCC should be performed using ultrasonography and patients should be screened at 6-month intervals (10). In Spain an important proportion of patients at risk of HCC are not in a surveillance program (11), which is due to a lack of strict compliance with the same, as well as not knowing of the existence of underlying liver cirrhosis in those patients with asymptomatic live disease.

Nowadays, the reference staging system is "The Barcelona Clinic Liver Cancer" (BCLC) staging system (1214). The main advantage of the BCLC staging system is that it links staging with treatment modalities and with an estimation of life expectancy that is based on published response rates to the different treatments (10). There is only one prospective trial globally validating the BCLC system (14). 
Currently alpha fetoprotein (AFP) is considered an inadequate screening or diagnostic test for $\mathrm{HCC}$ because of poor sensitivity and specificity. However, in some studies AFP was associated with survival $(15,16)$ and can help to identify subsets of HCC patients with increased risk for early recurrence and poor prognosis after hepatectomy (17) or transplant (18), but its value has not been properly defined.

The aim of this study was to evaluate the BCLC classification in our clinical practice and to know the prognostic value of the AFP.

\section{PATIENTS AND METHODS}

This observational study was approved by the Institutional Review Board of our hospital. Between January 2004 and November 2010, 136 consecutive HCC patients were prospectively included in this study. Only patients managed by specialized practitioners in HCC from our department were included. No patients were remitted to other hospitals, except those to be evaluated for LT (liver transplant). The patients were studied and managed according to usual clinical practice. The diagnosis of HCC was based on the recommendation of international guidelines: from 2001 we used the recommendation of the Barcelona-2000 European Association for the Study of the Liver Conference (19) and from 2005 the AASLD guidelines (20).

The following variables were recorded for each patient: age and sex of the patient, etiology of underlying liver disease, date and modality of HCC diagnosis, date of death or the latest follow-up visit, presence of cirrhosis (diagnosed with previous liver biopsy or unequivocal laboratory and imaging signs), liver tests and serum virological markers (determined by conventional methods), Child-Pugh stage and its constitutive variables (albumin, bilirubin, prothrombin time, ascites, encephalopathy), presence of other cancers and diabetes, tumor type, tumor extension, portal vein thrombosis, the AFP level (ng/dL) at diagnosis (evaluated by abdominal and thoracic CT), evidence of distant metastases at diagnosis, and treatment afforded. The presence of clinical relevant portal hypertension was based on presence of gastroesophageal varices, splenomegaly with platelet count less than $100,000 / \mathrm{mm}^{3}$ or hepatic venous pressure gradients higher than or equal to $10 \mathrm{mmHg}$.

Our surveillance program is based on ultrasound performed every 6 months. Serum AFP determination is also performed every 6 months. Staging of the HCC patients was performed using the BCLC system (12). Three patients with a single nodule greater than $8 \mathrm{~cm}$ were classified as stage B because we considered that they had a multinodular tumor.

The AFP was determined by electrochemiluminescence immunoassay (Roche Diagnostics ${ }^{\odot}$ ) with a Modular Analytics E170.

The decision for therapy was made by the specialist practitioner and in special cases made by a multidisciplinary team consisting of hepatologists, oncologists, radiologists and surgeons. In most cases we used therapeutic strategies according to the BCLC system: liver transplant (LT) in a reference center, liver resection (LR), percutaneous ethanol injection (PEI) and radiofrequency ablation (RF), transarterial chemo-embolization (TACE), sorafenib (from June 2009) and symptomatic treatment. When clinically indicated, we used a combined treatment.

Percutaneous ablation therapies (PEI and RF) were performed after intravenous analgesia and sedatives, as well as local anesthesia. PEI was performed under ultrasound guidance. The amount of ethanol used and the number of sessions depended on the size of the nodule. We perform RF from November 2007 with LeVeen needle electrode (Boston Scientific) according to the manufacturer's protocol. After completion of PEI or RF, a dynamic CT scan was performed. Complete response was defined as the absence of enhanced tumoral areas reflecting complete tissue necrosis. Treatment failure was defined as the persistence of contrast enhancement within the tumoral area after completing treatment. Treatment was repeated (PEI or RF or combination of both) if complete response was not achieved. In the last few years, before CT scan we usually performed a Contrast enhanced ultrasound (CEUS) with SonoVue ${ }^{\odot}$ (Bracco) and if there was viable tumor we repeated percutaneous treatment (21). TACE was performed with adriamicin plus lipiodol (until 2006) and the selective hepatic artery obstruction was achieved by the injection of polyvinyl alcohol particles $300-500 \mathrm{mcm}$ or microspheres, and from 2007, our group realize precision TACE, this embolization was performed using each of 300-500 and $500-700 \mathrm{mcm}$ vials of doxorubicin $(25 \mathrm{mg} / \mathrm{vial})$ saturated DCBead (Biocompatibles, Surrey, UK). Embolizations were as selective as possible.

Only mortality because of $\mathrm{CHC}$ or $\mathrm{CHC}$ and progressive deterioration of liver function were the only end-points used in the analysis. Survival was defined as the time elapsed from the date of diagnosis and either the date of death or the date of last follow-up information. We regularly phone the patients when they don't keep an appointment.

Univariate survival curves were estimated using the Kaplan-Meier method; the differences in the survival rates between the groups were compared using the log-rank test. The Cox proportional hazards model was used for the multivariate analysis. Statistical analysis was performed using SPSS 15 for Windows. All p values were derived from 2tailed tests and a level of $<0.05$ was accepted as statistically significant.

\section{RESULTS}

A total of 136 patients with $\mathrm{HCC}$ were analyzed in this study. $80.9 \%$ of patients were male. The mean age of the patients was $66.62 \pm 11.68$ years. Liver cirrhosis was present in $91.2 \%$. The most frequent cause of liver disease was hepatitis C infection (38.97\%), followed by alcohol (22.79\%), hepatitis B (11.03\%) and $14.72 \%$ being a mix (VHB and/or VHC) 
Table I. Survival rates according BCLC system

\begin{tabular}{llllc}
\hline \multicolumn{5}{c}{ Survival $(\% \pm E S)$} \\
\hline & $B C L C A$ & $B C L C B$ & $B C L C C$ & $B C L C D$ \\
\hline 6 months & $98,7 \pm 1,3$ & $72,7 \pm 8,8$ & $46,6 \pm 14,3$ & $18,2 \pm 11,6$ \\
12 months & $91,9 \pm 3,5$ & $53,8 \pm 10,5$ & $23,3 \pm 13,7$ & - \\
24 months & $75,9 \pm 6,3$ & $29,4 \pm 9,9$ & - & - \\
36 months & $59,1 \pm 8,4$ & $29,4 \pm 9,9$ & - & - \\
48 months & $59,3 \pm 8,4$ & $22 \pm 9,8$ & - & - \\
60 months & $52,6 \pm 9,7$ & $22 \pm 9,8$ & - & - \\
\hline
\end{tabular}

BCLC: The Barcelona Clinic Liver Cancer; SE: standard error. There were statistically significant differences in survival rates among each group $(p<0.0001)$.

and/or alcohol. Other less frequent causes were hemochromatosis $(2.94 \%)$, nonalcoholic fatty liver disease $(1.47 \%)$, primary biliary cirrhosis $(0.74 \%)$, porphyria $(1.47 \%)$ and cryptogenetic cirrhosis $(5.87 \%)$. There were 4 patients with positive serology for HIV. The $30.1 \%$ was diabetics and $10.3 \%$ had another tumor in the past.

A total of 86 patients $(63.2 \%)$ were diagnosed during the screening program. Non-invasive diagnosis of HCC (20) was performed in 100 patients (73.5\%). Most patients had Child-Pugh class A (71.3\%) or B (22.8\%), and only $5.9 \%$ had Child-Pugh class C. A total of 23 patients (16.9\%) had vascular invasion. A total of 6 patients $(4.4 \%)$ had extrahepatic spread. At diagnosis, serum AFP was $\leq 20 \mathrm{ng} / \mathrm{mL}$ in the $57 \%,>20-200 \mathrm{ng} / \mathrm{mL}$ in the $20 \%$ and $>200 \mathrm{ng} / \mathrm{mL}$ in $23 \%$. A patient was excluded from the analyses because AFP was not available at diagnosis.

According to BCLC staging system, 79 patients were classified as stage A $(58.09 \%)-12$ patients $(8.82 \%)$ were classified as stage 0 , very early-, $29(21.32 \%)$ stage B, 17 $(12.50 \%)$ stage $\mathrm{C}$ and 11 patients $(8.09 \%)$ as stage $\mathrm{D}$.

Initially several therapeutic strategies were used. LR was performed in 16 patients (11.76\%); RF in 14 patients (10.29\%), PEI in $31(22.79 \%)$, used as definitive treatments or as bridge therapies to transplant; TACE in 24 (17.65\%), sorafenib in $7(5.15 \%)$, and symptomatic treatment in 43 patients $(31.62 \%)(22.06 \%$ because there were no options and $9.56 \%$ rejected the offered treatment). LT was performed in 9 patients $(6.62 \%), 8$ of whom were treated before transplant with another therapy while on the waiting list. After the initial treatment the patients were treated according to their new clinical situation. When there was progression of the tumor the patients were treated according to the new stage.

Twenty of 79 patients in stage A (including stage 0 ) of BCLC $(25.32 \%)$ were treated with no curative treatments due to several reasons linked to the potential limits of the applicability of the algorithm. Of these 20 patients, 11 patients rejected the initial offered treatment and 9 patients were treated by TACE as it was the only technical possibility. The rest of stage A patients were treated initially: 15 with LR, 29 with PEI, 14

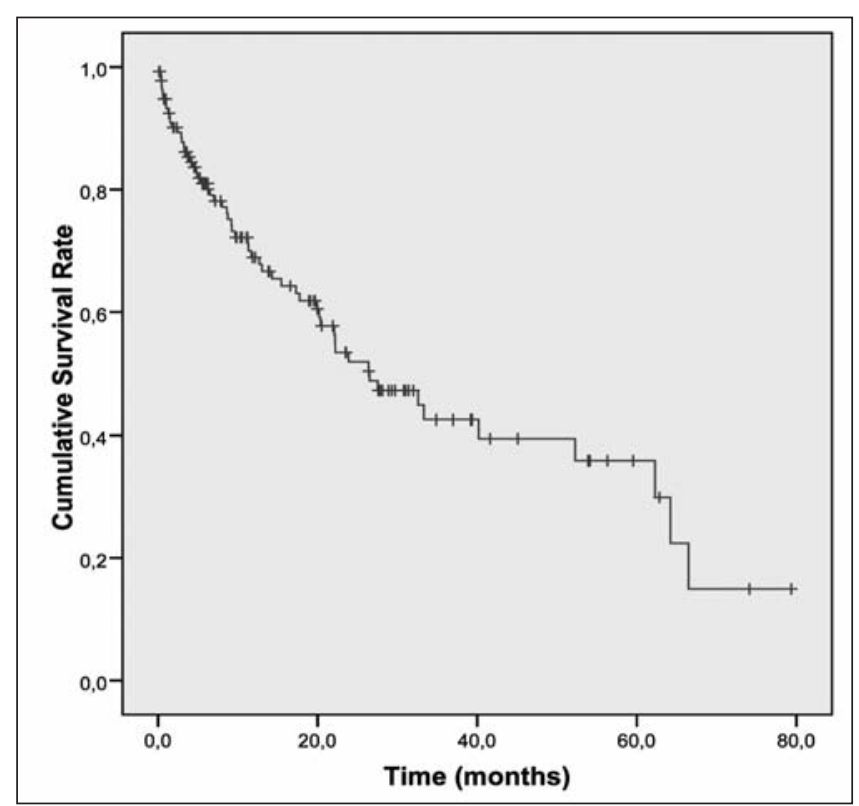

Fig. 1. Overall survival. The overall median survival time was 26.52 months (95\% Cl 16.7-36.3).

with RF and 1 with LT without bridge treatment. Symptomatic treatment was the only option for treatment in 10 patients in stage B of BCLC (34.5\%). Two patients initially treated with PEI and one with LR in the end were classified as stage B after reevaluation.

The mean follow-up period of all patients was $16.99 \pm$ 17.58 months. During the follow-up period, 59 patients (43.38\%) died and 8 patients were lost to follow-up. The overall median survival time was 26.52 months (95\% CI 16.7- 36.3). The 1, 2, 3, 4 and 5 year survival rate was $68.9 \%$, $51.9 \%, 42.5 \%, 39.5 \%$ and $35.9 \%$, respectively (Fig. 1).

Figure 2 illustrates the survival curves of HCC patients stratified according to the BCLC classification. There were statistically significant differences in survival rates among each group $(\mathrm{p}<0.0001)$ (Table I). The median survival among each group was: BCLC A 62.27; BCLC B 12.72; BCLC C 4.83, and BCLC D 0.62 months.

Table II illustrates the survival rates according to serum AFP (AFP $\leq 20,>20-200$ and $>200 \mathrm{ng} / \mathrm{mL}$ ). The median survival among each group was: AFP $\leq 20$ : 62.27 months; > 20-200: 22.08 months and > $200 \mathrm{ng} / \mathrm{mL}$ : 5.39 months $(\mathrm{p}<0.0001)$. The figure 3 shows the survival curves according the serum AFP.

The Cox model was used to identify simple independent predictors of survival. Whether the patient was treated or not was included in the model as in some cases patients could not be treated according the BCLC system (no options o rejected treatment). Multivariate analysis showed, after adjusting for age, sex and treatment, that AFP, BCLC classification and treatment were independent prognostic factors, reducing the risk of death in treated patients and increasing it in groups B, C and D of the BCLC classification (Table III). Furthermore, the risk of death increased as 
Table II. Survival rates according serum AFP

Survival $(\% \pm E S)$

AFP $0-20 \mathrm{ng} / \mathrm{ml} \quad$ AFP $>20-200 \mathrm{ng} / \mathrm{ml} \quad$ AFP $>200 \mathrm{ng} / \mathrm{ml}$

\begin{tabular}{lccc}
\hline 12 months & $83,7 \pm 4,5$ & $69,3 \pm 10,6$ & $27,2 \pm 9,3$ \\
24 months & $68,5 \pm 6,4$ & $43,2 \pm 12,3$ & $10,2 \pm 8,6$ \\
36 months & $59,1 \pm 7,6$ & $27 \pm 12$ & $10,2 \pm 8,6$ \\
48 months & $54,2 \pm 8,4$ & $27 \pm 12$ & - \\
60 months & $54,2 \pm 8,4$ & - & -
\end{tabular}

There were statistically significant differences in survival rates among each group $(p<0.0001)$. AFP: alpha-fetoprotein; SE: standard error.

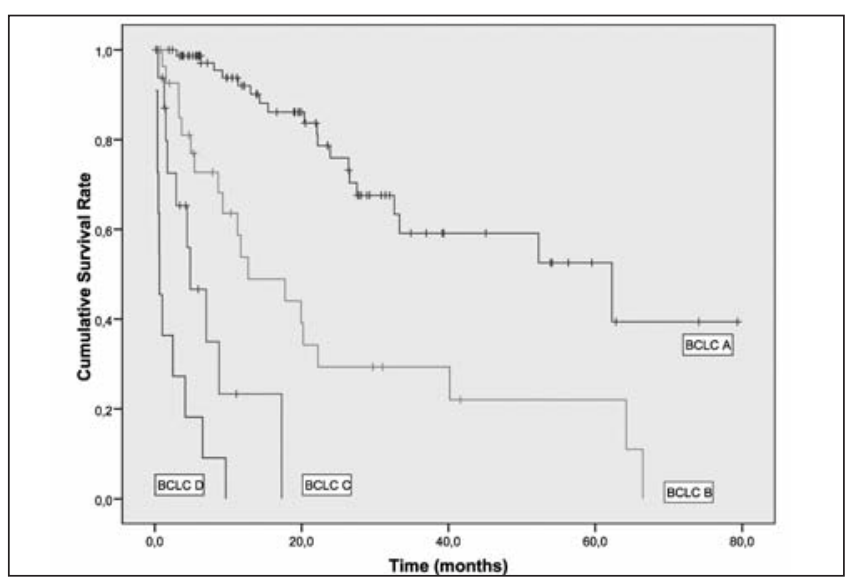

Fig. 2. Survival according BCLC system. There were statistically significant differences in survival rates among each group $(p<0.0001)$. BCLC: The Barcelona Clinic Liver Cancer.

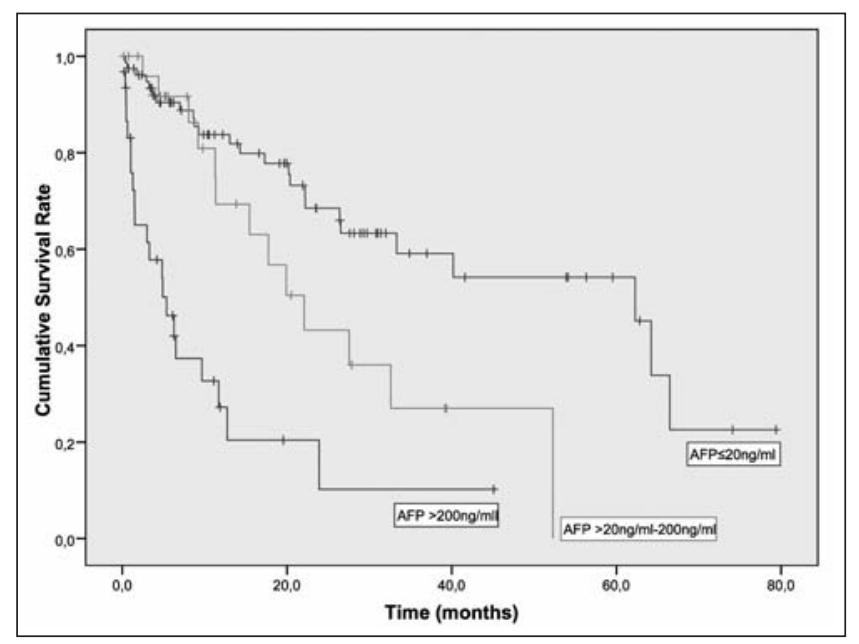

Fig. 3. Survival curves according AFP. There were statistically significant differences in survival rates among each group ( $p<0.0001)$. AFP: alphafetoprotein

the concentration of AFP increased, with a hazard ratio (HR) of 2.161 (CI 95\%: 1.003-4.656) and of 2.944 (CI 95\%: 1.415-6.127) in groups with intermediate and upper level AFP, $>20-200$ and $>200 \mathrm{ng} / \mathrm{mL}$ respectively, in relation to groups with lower level AFP $(\leq 20)$.

\section{DISCUSSION}

Over the past few years, the wide spread use and development of more sophisticated diagnostic tools, and the improvement of therapeutic techniques, although not "rad-

Table III. Multivariate analysis

\begin{tabular}{|c|c|c|c|c|}
\hline & $p$ & $H R$ & IC 95\% & \\
\hline & & & Lower & Upper \\
\hline $\mathrm{AFP} \leq 20 \mathrm{ng} / \mathrm{ml}$ & 0,009487 & & & \\
\hline AFP > $20-200 \mathrm{ng} / \mathrm{ml}$ & 0,049192 & 2,16072254 & 1,00272929 & 4,65601432 \\
\hline AFP $>200 \mathrm{ng} / \mathrm{ml}$ & 0,003870 & 2,9443783 & 1,4150306 & 6,12662621 \\
\hline$B C L C A$ & 0,000001 & & & \\
\hline BCLC B & 0,000188 & 3,70430383 & 1,86304591 & 7,36528649 \\
\hline $\mathrm{BCLC} C$ & 0,000001 & 11,9386479 & 4,39502302 & 32,430163 \\
\hline$B C L C D$ & 0,000001 & 15,0476799 & 5,045057 & 44,8820837 \\
\hline Standard treatment* & 0,001284 & 0,32176942 & 0,16134006 & 0,6417226 \\
\hline Age $\geq 65$ años & 0,696517 & 0,88032001 & 0,46392516 & 1,67044898 \\
\hline Sex (male) & 0,8596877 & 0,92796402 & 0,40507108 & 2,12584226 \\
\hline
\end{tabular}

AFP, BCLC classification and treatment were independent prognostic factors. *Standard treatment: liver resection, liver transplant, percutaneous ethanol injection, radiofrequency ablation, transarterial chemo-embolization or sorafenib. HR: hazard ratio; BCLC: The Barcelona Clinic Liver Cancer; AFP: alpha-fetoprotein. 
ical", have allowed a higher number of HCC patients to be treated and have led to an improvement in overall survival. Therefore, the management of HCC patients has changed because of an improvement in diagnosis, patient care and treatment outcomes. As such, it is necessary to improve the prediction of the prognosis of these patients in order to properly identify the potential candidates for therapy and to critically evaluate the results of new treatment modalities.

Many studies have reported variable survival in $\mathrm{HCC}$ patients. A meta-analysis (22) in untreated HCC patients confirms the heterogeneity of behavior of HCC. Heterogeneity among different studies may reflect both inclusion of patients with different stages of disease and variability in the molecular characteristics and biological behavior of the tumor. There are also many potential biases in the selection of patients with different demographic and clinical characteristics, different timing of referral and diagnostic criteria, severity of the underlying cirrhosis, and tumor burden in terms of number and size of HCC nodules and of presence of macrovascular invasion or extrahepatic spread. And some studies included special patients, for example only asymptomatic patients with non-surgical treatment. In retrospective studies a selection bias could have occurred, with a reduced prevalence of patients with a worse prognosis. All these factors contribute to difficulty of comparing the different studies.

The 1 and 3 year survival rates reported in past years, including treated patients, range from 44.3 to $67 \%$ and 24 to $45 \%$, respectively $(14,23-30)$. The median survival ranges from 8 to 37.7 months (14,23-26,28,30-33). However, we must consider all potential biases in the selection of patients with different stages, demographic and clinical characteristics. In our study, the overall median survival time was 26.52 months and the 1, 2, 3, 4 and 5 year survival rate was $68.9 \%, 51.9 \%, 42.5 \%, 39.5 \%$ and $35.9 \%$, respectively (Fig. 1). In this sense, we think our series is possibly more homogeneous and more appropriately reflects the evolution of HCC patients in a hospital such as ours, due to less biases by including all HCC patients in our department, and by not excluding patients with worse prognosis who normally would not be included in series from reference hospitals.

The Okuda staging system was the first system to incorporate liver function status into the staging of HCC (34). However, this system is useful for identifying only endstage patients, and provides no valid discrimination for less advanced patients. Several additional investigations to identify variables related to prognosis in many countries have been published in the last years. The BCLC staging system was developed based on the combination of data from several independent studies representing different disease stages and/or treatment modalities and has been externally validated $(26,35)$, although there is only a prospective trial (14). In our study we also validated the BCLC as a good prognostic system. Currently, it is de facto reference staging system. According the BCLC system the survival varies from one study to another. In a meta-analysis study of untreated patients the pooled estimated of BCLC B stage 1-year survival rate was $49.6 \%$, BCLC C $25 \%$ and BCLC D $11 \%$ (22). In treated patients, Cillo (14) reported a 53 months median survival in the BCLC stage A, 16 months in BCLC B, 7 months in BCLC C and 3 months in BCLC D. In the report of Grieco (35), the median survival was 22.3 to 43.4 months in the BCLC stage A, 17.6 months in the BCLC B and 7.4 months in the BCLC stage C. Series of untreated intermediate HCC showed a 1-year survival of $80 \%$ and for advanced HCC of $29 \%$ (24). We had similar 1 and 3-year survival rate as Cillo (14), although in those with more advanced illness (stage B-D), our patients had a slightly worse prognosis. As we have said before, our series is possibly more homogeneous in terms of diagnosis, staging and treatment.

There are other staging systems. In Japanese patients, the stratification ability and prognostic predictive power of the Japan Integrated Score (JIS) were much better than that of the Cancer of Liver Italian Program (CLIP) score and were simple to obtain and remember $(36,37)$. In a retrospective study in Japan, the JIS system proved to be more suitable than CLIP and BCLC (38). In the Yen study (16), the CLIP and a modified TNM-based Japan Integrated Staging (JIS) score were found to be superior to the BCLC system for the prediction of prognosis of HCC patients. In contrast, the BCLC system has recently been validated in American (26) and Italian (14) cohorts, and was shown to be of better predictive power for survival than other staging systems for HCC. In fact, in the west the BCLC stage system is the most used, with our results confirming that BCLC is a good prognostic system.

The prognostic value of AFP has not been properly defined. In some studies AFP was associated with survival $(23,28-32)$ but in others it did not define practical value $(24,39)$. A systematic review showed that the most robust predictors of death were portal vein thrombosis, tumor size, AFP and Child-Pugh class (30). In this review, the AFP was among the first five significant variables in 20 studies. In one study, the AFP was an independent risk factor for mortality only in untreated patients (40). Some studies found that HCC patients with high AFP tended to have greater tumor size, bilobar involvement, massive or diffuse types, and portal vein thrombosis, and equally, AFP was a prognostic indicator (41). Farinati (39) confirmed the prognostic value of AFP in a study with 1,158 patients, with poorer survival in patients with higher levels, but no clear prognostic impact in the individual patient. In opinion of Yen, the prognostic predictive power of AFP could not be replaced by tumor factor and liver function reserve factor and the modified TNM based JIS system combined with AFP levels may now be the most applicable system (16).

AFP elevation, more than a coincidental epiphenomenon, appears to contribute to vascular invasion and HCC progression and help to identify subsets of HCC patients with increased risk for early recurrence and poor prognosis after hepatectomy (17). Others studies also found a biologic role 
of AFP $(39,42)$. High AFP may contribute to the metastatic potential and tumor progression and is an important unfavorable prognostic factor for $\mathrm{HCC}$ after tumor resection (17). In a study the AFP and the tumor volume predicted HCC recurrences after LT, and they may improve the selection of patients (18). In this respect, one study showed that patients treated with PEI with a pretreatment AFP level of $200 \mathrm{ng} / \mathrm{ml}$ or less had a better prognosis than patients with an AFP level higher than $200 \mathrm{ng} / \mathrm{ml}$ (43). Therefore, we thought it would be interesting to see whether the AFP adds to the prognostic value of the BCLC. We found AFP was an independent prognostic factor (Table III), and we think it can improve the BCLC system. In this way, in the last years there were improvements in the different prognostic scores. In fact, the new editions of the staging systems appear to have better prognostic stratification than older ones $(27,33,44)$ and we think AFP can be a factor in the improvement of the BCLC system, although, we cannot say for sure how, due to the low number of patients in our study. Perhaps, an improved BCLC system would be able to show that patients in BCLC B stage with normal AFP could have a similar prognosis to those with BCLC A with a higher AFP, modifying the management of these patients. It could also aid in the management of HCC patients on waiting list or in the election of the best possible treatment for those patients. In our opinion, the effectiveness of more powerful prognostic criteria such as tumor size, vascular invasion, Child class and extrahepatic spread can be improved by the prognostic value of AFP.

In summary, our results confirm that BCLC is a good prognostic system and that AFP has prognosis value in $\mathrm{HCC}$ patients. The addition of AFP could improve the BCLC system, although future studies are needed to confirm our results and to know the best way to combine BCLC and AFP.

\section{ACKNOWLEDGMENTS}

The authors wish to thank Rafael Cuena for his support with the statistics.

\section{REFERENCES}

1. El-Serag HB, Lenhard Rudolph K. Hepatocellular carcinoma: epidemiology and molecular carcinogenesis. Gastroenterology 2007;132:2557-76.

2. Benvegnu L, Gios M, Boccato S, Alberti A. Natural history of compensated viral cirrhosis: a prospective study on the incidence and hierarchy of major complications. Gut 2004; 53:7449.

3. Fattovich G, Stroffolini T, Zagni I, Donato F. Hepatocellular carcinoma in cirrhosis: incidence and risk factors. Gastroenterology 2004;127:S35S50.

4. Bosetti C, Levi F, Boffetta P, Lucchini F, Negri E, La Vecchia C. Trends in mortality from hepatocellular carcinoma in Europe, 1980-2004. Hepatology 2008;48:137-45.

5. Capocaccia R, Sant M, Berrino F, Simonetti A, Santi V, Trevisani F, et al. Hepatocellular carcinoma: trends of incidence and survival in Europe and the United States at the end of the 20th century. Am J Gastroenterol 2007;102:1-10.
6. Yu MC, Yuan JM. Environmental factors and risk for hepatocellular carcinoma. Gastroenterology 2004;127:S72-S78.

7. Bolondi L. Screening for hepatocellular carcinoma in cirrhosis. J Hepatol 2003;39:1076-84.

8. Zhang BH, Yang BH, Tang ZY. Randomized controlled trial of screening for hepatocellular carcinoma. J Cancer Res Clin Oncol 2004;130:417-22.

9. Sangiovanni A, Del Ninno E, Fasani P, de Fazio C, Ronchi G, Romeo R. Increased survival of cirrhotic patients with a hepatocellular carcinoma detected during surveillance. Gastroenterology 2004;126:100514 .

10. Bruix J, Sherman M. Management of hepatocellular carcinoma: an update. Hepatology 2011;53:1020-2.

11. Varela M, Reig M, de la Mata M, Matilla A, Bustamante J, Pascual S, et al. Treatment approach of hepatocellular carcinoma in Spain. Analysis of 705 patients from 62 centers. Med Clin (Barc) 2010;134:569-76.

12. Llovet JM, Brú C, Bruix J. Prognosis of hepatocellular carcinoma: the BCLC staging classification. Seminar in Liver Disease 1999;19:32938.

13. Bruix J, Llovet JM. Prognostic prediction and treatment strategy in hepatocellular carcinoma. Hepatology 2002;35:519-24.

14. Cillo U, Vitale A, Grigoletto F, Farinati F, Brolese A, Zanus G, et al. Prospective validation of the Barcelona Clinic Liver Cancer staging system. J Hepatol 2006;44:723-31.

15. Trevisani F, Magini G, Santi V, Morselli-Labate AM, Cantarini M Ch, Di Nolfo MA, et al. Impact of etiology of cirrhosis on the survival of patients diagnosed with hepatocellular carcinoma during surveillance. Am J Gastroenterol 2007;102:1022-31.

16. Yen YH, Changchien CS, Wang JH, Kee KM, Hung CH, Hu TH, et al. A modified TNM-based Japan Integrated Score combined with AFP level may serve as a better staging system for early-stage predominant hepatocellular carcinoma patients. Dig Dis 2009;41:431-41.

17. Peng SY, Chen WJ, Lai PL, Jeng YM, Sheu JC, Hsu HC. High -fetoprotein level correlates with high stage, early recurrence and poor prognosis of hepatocellular carcinoma: significance of hepatitis virus infection, age, p53 and -catenin mutations. Int J Cancer 2004;112:44-50.

18. Ravaioli M, Ercolani G, Cescon M, Vetrone G, Voci C, Grigioni WF, et al. Liver transplantation for hepatocellular carcinoma: further considerations on selection criteria. Liver Transplantation 2004;10:1195202.

19. Bruix J, Sherman M, Llovet JM, Beaugrand M, Lencioni R, Burroughs AK, et al. clinical management of hepatocellular carcinoma. Conclusions of the Barcelona-2000 EASL Conference. J Hepatol 2001;35:42140.

20. Bruix J, Sherman M. Management of hepatocellular carcinoma. Hepatology 2005;42:1208-36.

21. Nicolau C, Vilana R, Bianchi L, Bru C. Early-stage hepatocellular carcinoma: the high accuracy of real-time contrast-enhanced ultrasonography in the assessment of response to percutaneous tratment. Eur Radiol 2007;17(Supl. 6):F80-F88.

22. Cabibbo G, Enea m, Attanasio M, Bruix j, Craxi A, Gammà C. A metaanalysis of survival rates of untreated patients in randomized clinical trials of hepatocellular carcinoma. Hepatology 2010;51:1274-83.

23. The Cancer of the Liver Italian Program (CLIP) Investigators. A new prognostic system for hepatocellular carcinoma: a retrospective study of 435 patients. Hepatology 1998;28:751-5.

24. Llovet JM, Bustamante J, Castells A, Vilana R, Ayuso MC, Sala M, et al. Natural history of untreated nonsurgical hepatocellular carcinoma: rationale for the design and evaluation of therapeutic trials. Hepatology 1999;29:62-7.

25. Cillo U, Bassanell M, Vitale A, Grigoletto A, Burra P. Fagiuoli S, et al. The critical issue of hepatocellular carcinoma prognostic classification: which is the best tool available? J Hepatol 2004;40:124-31.

26. Marrero JA, Fontana RJ, Barrat A, Askari F, Conjeevaram HS, Su GL, et al. Prognosis of hepatocellular carcinoma: comparison of 7 staging systems in an American cohort. Hepatology 2005;41:707-16.

27. Kee K-M, Wang J-H, Lee Ch-M, Chen Ch-L, Changchien Ch-S, Hu T-H, et al. Validation of clinical AJCC/UICC TNM staging system for hepatocellular carcinoma: Analysis of 5,613 cases from a medical center in southern Taiwan. Int J Cancer 2007;120:2650-5.

28. Park KW, Park J-W, Choi J, Kim TH, Kim SH, Park HS, et al. Survival analysis of 904 patients with hepatocellular carcinoma in a hepatitis B virus-endemic area. J Gastroen Hepatol 2007;23:467-73. 
29. Changchien Ch-S, Chen Ch-L, Yen Y-H, Wang J-H, Hu T-H, Lee Ch$\mathrm{M}$, et al. Analysis of 6381 hepatocellular carcinoma patients in southern Taiwan: prognostic features, treatment outcome, and survival. J Gastroenterol 2008;43:159-70.

30. Tandon P, Garcia-Tsao G. Prognostic indicators in hepatocellular carcinoma: a systematic review of 72 studies. Liver Int 2009;29:502-10.

31. Schöinger-Hekele M, Müler C, Kutilek M, Oesterreicher C, Ferenci P, Gangl A. Hepatocellular carcinoma in Central Europe: prognostic features and survival. Gut 2001;48:103-9.

32. Kuo Y-H, Lu Sh-N, Chen Ch-L, Cheng Y-F, Lin Ch-Y, Hung Ch-H, et al. Hepatocellular carcinoma surveillance and appropriate treatment options improve survival for patients with liver cirrhosis. Eur J Cancer 2010;46:744-51.

33. Ueno Sh, Tanabe G, Nuruki K, Hamanoue M, Komorizono Y, Oketani $\mathrm{M}$, et al. Prognostic performance of the new classification of primary liver cancer of Japan (4th edition) for patients with hepatocellular carcinoma: a validation analysis. Hepatol Res 2002;24:395-403.

34. Okuda K, Ohtsuki T, Obata H, Tomimatsu M, Okazaki N, Gasegawa $\mathrm{H}$, et al. Natural history of hepatocellular carcinoma and prognosis in relation to treatment. Study of 850 patients. Cancer 1985;56:918-28.

35. Grieco A, Pompili M, Caminiti G, Miele L, Covino M, Alfei B, et al. Prognostic factors for survival in patients with early-intermediate hepatocellular carcinoma undergoing non-surgical therapy: comparison of Okuda, CLIP, and BCLC staging systems in a single Italian centre. Gut 2005;54:411-8.

36. Kudo M, Chung H, Osaki Y. Prognostic staging system for hepatocellular carcinoma (CLIP score): its value and limitations, and a proposal for a new staging system, the Japan Integrated Staging Score (JIS score). J Gastroenterol 2003;38:207-15.
37. Kudo M, Chung H, Haji S, Osaki Y, Oka H, Seki T, et al. Validation of a new prognostic staging system for hepatocellular carcinoma: the JIS Score compared with the CLIP Score- Hepatology 2004;40:1396-405.

38. Toyoda H, Kumada T, Kiriyama S, Sone Y, Tanikawa M, Hisanaga $\mathrm{Y}$, et al. Comparison of the usefulness of three staging systems for hepatocellular carcinoma (CLIP, BCLC, and JIS) in Japan. Am J Gastroenterol 2005;100:1764-71.

39. Farinati F, Marino D, De Giorgio M, Baldan A, Cantarini M, Cursaro C, et al. Diagnostic and prognostic role of -fetoprotein in hepatocellular carcinoma: Both or Neither? Am J Gastroenterol 2006;101:524-32.

40. Cammà C, Di Marco V, Cabibbo G, Latteri F, Sandonato L, Parisi $\mathrm{P}$, et al. Survival of patients with hepatocellular carcinoma in cirrhosis: a comparison of BCLC, CLIP and GRETCH staging systems. Aliment Pharmacol Ther 2008;28:62-75.

41. Tangkijvanich P, Anukulkarnkusol N, Suwangool P, Lertmaharit S, Hanvivatvong $\mathrm{O}$, Kullavanijaya $\mathrm{P}$, et al. Clinical characteristics and prognosis of hepatocellular carcinoma. Analysis based on serum alphafetoprotein levels. J Clin Gastroenterol 2000;31:302-8.

42. Peng Sh-Y, Lai P-L, Chu J-Sh, Lee P-H, Tsung P-T, Chen D-Sh, et al. Expression and hypomethylation of alpha-fetoprotein gene in unicentric and multicentric human hepatocellular carcinoma. Hepatology 1993;17:35-41.

43. Lencioni R, Bartolozzi C, Caramella D, Paolicchi A, Carrai M, Maltini $\mathrm{G}$, et al. Treatment of small hepatocellular carcinoma with percutaneous ethanol injection. Cancer 1995;76:1737-46.

44. Huo T-I, Huang Y-H, Lin H-Ch, Wu J-Ch, Chiang J-H, Lee P-Ch, et al. Proposal of a modified cancer of the liver italian program staging system based on the model for end-stage liver disease for patients with hepatocellular carcinoma undergoing loco-regional therapy. Am J Gastroenterol 2006;101:975-82. 